\title{
Characterizing the heterogeneity in 5-aminolevulinic acid-induced fluorescence in glioblastoma
}

\author{
Damian A. Almiron Bonnin, PhD, ${ }^{1,2}$ Matthew C. Havrda, PhD, ${ }^{1,2}$ Myung Chang Lee, BA, ${ }^{2,3}$ \\ Linton Evans, MD, ${ }^{4,5}$ Cong Ran, MD, PhD, ${ }^{1,2}$ David C. Qian, MD, PhD, ${ }^{6}$ Lia X. Harrington, PhD, ${ }^{6}$ \\ Pablo A. Valdes, MD, PhD, ${ }^{7}$ Chao Cheng, PhD, ${ }^{1,2,6}$ Chris I. Amos, PhD, ${ }^{1,2,6}$ Brent T. Harris, MD, PhD, ${ }^{8}$ \\ Keith D. Paulsen, PhD, ${ }^{2,4,5,9}$ David W. Roberts, MD,2,4,5,9 and Mark A. Israel, MD1,2,10,11 \\ ${ }^{1}$ Department of Molecular and Systems Biology, Geisel School of Medicine at Dartmouth, Hanover; ${ }^{2}$ Norris Cotton Cancer \\ Center, Geisel School of Medicine at Dartmouth, Lebanon; ${ }^{3}$ Department of Biology, Dartmouth College, Hanover; ${ }^{4}$ Department of \\ Surgery (Neurosurgery), Geisel School of Medicine at Dartmouth, Hanover; ${ }^{5}$ Department of Neurosurgery, Dartmouth-Hitchcock \\ Medical Center, Lebanon; ${ }^{\circ}$ Department of Biomedical Data Science, Geisel School of Medicine at Dartmouth, Hanover, New \\ Hampshire; 'Department of Neurosurgery, Brigham and Women's Hospital, Harvard Medical School, Boston, Massachusetts; \\ ${ }^{8}$ Department of Pathology, Georgetown University Medical Center, Washington, DC; ${ }^{9}$ Thayer School of Engineering, Dartmouth \\ College, Hanover; ${ }^{10}$ Department of Medicine, Geisel School of Medicine at Dartmouth, Hanover; and ${ }^{11}$ Department of Pediatrics, \\ Geisel School of Medicine at Dartmouth, Hanover, New Hampshire
}

OBJECTIVE 5-aminolevulinic acid (5-ALA)-induced protoporphyrin IX (PpIX) fluorescence is an effective surgical adjunct for the intraoperative identification of tumor tissue during resection of high-grade gliomas. The use of 5-ALA-induced PpIX fluorescence in glioblastoma (GBM) has been shown to double the extent of gross-total resection and 6-month progression-free survival. The heterogeneity of 5-ALA-induced PpIX fluorescence observed during surgery presents a technical and diagnostic challenge when utilizing this tool intraoperatively. While some regions show bright fluorescence after 5-ALA administration, other regions do not, despite that both regions of the tumor may be histopathologically indistinguishable. The authors examined the biological basis of this heterogeneity using computational methods.

METHODS The authors collected both fluorescent and nonfluorescent GBM specimens from a total of 14 patients undergoing surgery and examined their gene expression profiles.

RESULTS In this study, the authors found that the gene expression patterns characterizing fluorescent and nonfluorescent GBM surgical specimens were profoundly different and were associated with distinct cellular functions and different biological pathways. Nonfluorescent tumor tissue tended to resemble the neural subtype of GBM; meanwhile, fluorescent tumor tissue did not exhibit a prominent pattern corresponding to known subtypes of GBM. Consistent with this observation, neural GBM samples from The Cancer Genome Atlas database exhibited a significantly lower fluorescence score than nonneural GBM samples as determined by a fluorescence gene signature developed by the authors.

CONCLUSIONS These results provide a greater understanding regarding the biological basis of differential fluorescence observed intraoperatively and can provide a basis to identify novel strategies to maximize the effectiveness of fluorescence agents.

https://thejns.org/doi/abs/10.3171/2019.2.JNS183128

KEYWORDS glioblastoma; 5-aminolevulinic acid; tumor heterogeneity; fluorescence-guided surgery; oncology

$\mathrm{G}$ Lioblastoma (GBM) is the most aggressive and common form of primary brain tumor in adults, with a median patient survival of less than 2 years despite aggressive multimodal treatment that includes maximal resection, chemotherapy, radiation therapy, and, most recently, tumor-treating fields..$^{15,30,31}$ Studies have demonstrated that maximizing the extent of tumor resection is a significant modifiable factor that improves overall survival. ${ }^{5}$ However, the surgeon's ability to achieve a gross-total resection (GTR) is challenged by the lack of discernible tumor margins, given the diffusely infiltrative nature of GBM. ${ }^{45}$ The use of 5-aminolevulinic acid

ABBREVIATIONS GBM = glioblastoma; GSEA = Gene Set Enrichment Analysis; GTR = gross-total resection; IPA = Ingenuity Pathway Analysis; PC = principal component; $\mathrm{PCA}=\mathrm{PC}$ analysis; PpIX = protoporphyrin IX; RMA = robust multichip analysis; TCGA = The Cancer Genome Atlas; 5-ALA = 5-aminolevulinic acid.

SUBMITTED November 11, 2018. ACCEPTED February 22, 2019.

INCLUDE WHEN CITING Published online May 24, 2019; DOI: 10.3171/2019.2.JNS183128. 
(5-ALA)-induced protoporphyrin IX (PpIX) fluorescence has been shown to almost double the rates of GTR and 6-month progression-free survival in patients with GBM. ${ }^{10,29} 5$-ALA is an endogenous precursor in the heme biosynthesis pathway. ${ }^{21}$ When administered to individuals prior to surgery, the nonfluorescent prodrug 5-ALA leads to overproduction and preferential accumulation of the fluorescent molecule PpIX in malignant or neoplastic cells. ${ }^{21}$ The surgeon can use a surgical microscope modified for fluorescence imaging to illuminate the surgical field with violet-blue light, and tumor tissues with sufficient PpIX will demonstrate a visible red-pink fluorescence and normal tissues will not. ${ }^{21}$

The effectiveness of 5-ALA-induced PpIX fluorescence during resection ${ }^{10,29}$ is compromised by the observed heterogeneity of tumor fluorescence, such that tumor tissues with histopathologically indistinguishable features will demonstrate different fluorescence characteristics from bright fluorescence to no fluorescence. . $^{16,25,36,37}$ Tissue fluorescence was identified as bright red fluorescence or no fluorescence, in which the latter is categorized as likely not containing tumor. 5-ALA-induced PpIX fluorescence has a high positive predictive value, such that tissue with visible fluorescence is most likely to contain malignant cells. ${ }^{36,37}$ Nevertheless, this technique has demonstrated a low negative predictive value, in that tissue that does not display visible red fluorescence may or may not contain significant amounts of tumor. ${ }^{25,36}$ We have previously demonstrated the utility of quantitative technologies to help improve sensitivity and detection of lower levels of fluorescence by accounting for the varying effects of tissue optical properties. ${ }^{35,37,40}$ Nevertheless, this prior work did not explore, at a biological level, why some histopathologically indistinguishable tumor tissues produce higher levels of PpIX and others do not. In this study, we found that the gene expression profiles characterizing fluorescent and nonfluorescent GBM surgical specimens were profoundly different and were associated with distinct cellular functions and different biological pathways.

\section{Methods}

\section{Patient Tumor Sample Collection}

Tissue samples were collected from patients who were part of a larger investigational study to correlate 5-ALAinduced fluorescence, neuroimaging, and histopathology in patients undergoing volumetric resection of intracranial tumor (clinical trial registration no. NCT00870779, clinicaltrials.gov). Both the larger study and this study were approved by the Institutional Review Board for the Protection of Human Subjects, and informed consent was obtained from all patients. 5-ALA was used under an FDA Investigational New Drug approval.

Patients received 5-ALA orally $(20 \mathrm{mg} / \mathrm{kg}$ bodyweight, dissolved in $50 \mathrm{~mL}$ water; DUSA Pharmaceuticals) 3 hours prior to the induction of anesthesia. Tissue to be collected as a study specimen was graded in situ for visible fluorescence (no, low, moderate, or high fluorescence) by the surgeon (D.W.R.) under violet-blue light illumination using a Zeiss Pentero operating microscope modified for fluorescence guidance (Blue 400 fluorescence imaging module, Carl Zeiss Surgical GmbH). Specimens in this study had fluorescence grades of either no fluorescence or moderate/high fluorescence. Biopsies of sampled sites were divided into portions placed in formalin for histopathology or flash frozen and stored at $-80^{\circ} \mathrm{C}$ for subsequent microarray analysis (Fig. 1).

\section{Histopathology}

Tissues were formalin fixed, paraffin embedded, and stained with $\mathrm{H} \& \mathrm{E}$. Each $\mathrm{H}$ \& E-stained tissue section was assessed based on WHO histopathological criteria by a neuropathologist (B.T.H.) as previously described. ${ }^{25}$

\section{Microarray Analysis}

Microarray analysis was conducted by the Dartmouth Genomics Shared Resource. Total RNA was purified using the TRIzol method and labeled using the Illumina TotalPrep kit protocol or Affymetrix WT Plus kit per manufacturer's guidelines, and probed using the HumanHT-12 v4 BeadChip (Illumina) for the discovery study or the Clariom S human assay (Affymetrix) for the validation study. Raw data generated from the HumanHT-12 v4 BeadChip were processed in the BRB Array Tools Software using Variance Stabilizing Transformation followed by Robust Spline Normalization. Raw data generated from Clariom $\mathrm{S}$ mouse arrays were processed using Affymetrix Expression Console Software. CEL files containing feature intensity values were converted into summarized expression values by robust multichip analysis (RMA), which consists of a background adjustment, quantile normalization, and summarization across all chips. All samples passed quality control thresholds for hybridization, labeling, and the expression of housekeeping gene controls.

\section{Bioinformatics and Statistical Analysis}

To generate the list of differentially expressed genes (Supplementary Tables 1 and 2), we RMA normalized the microarray expression data of fluorescent and nonfluorescent specimens and converted the data to $\log 2$ values. To obtain the $\log 2$ fold change values, we averaged fluorescent and nonfluorescent specimen values for each gene, and we used the Student t-test to determine statistical significance. The volcano plot was generated using R ( $\mathrm{r}-$ project.org). The fold changes and associated $\mathrm{p}$ values of the differential gene expression between fluorescent and nonfluorescent samples were exported using $\mathrm{R}$ for further analysis by Ingenuity Pathway Analysis (IPA). ${ }^{27}$ Further statistical analyses and heat maps were performed with GraphPad Prism version 7.02 for Windows. Gene Set Enrichment Analysis (GSEA) was performed as previously described utilizing the GO and BIOCARTA gene sets indicated in Supplementary Fig. 1A-F., 3, 24,

To perform the principal component analysis (PCA), the expression levels of fluorescent and nonfluorescent samples from 10 patients were transformed into PCs using the R package FactoMineR.${ }^{19}$ Centroid values of the genes corresponding to the classical, mesenchymal, neural, and proneural subtypes of GBM were downloaded from https://tcga-data.nci.nih.gov/docs/publications/gbm_exp/ and separately transformed into PCs. We plotted the top 3 

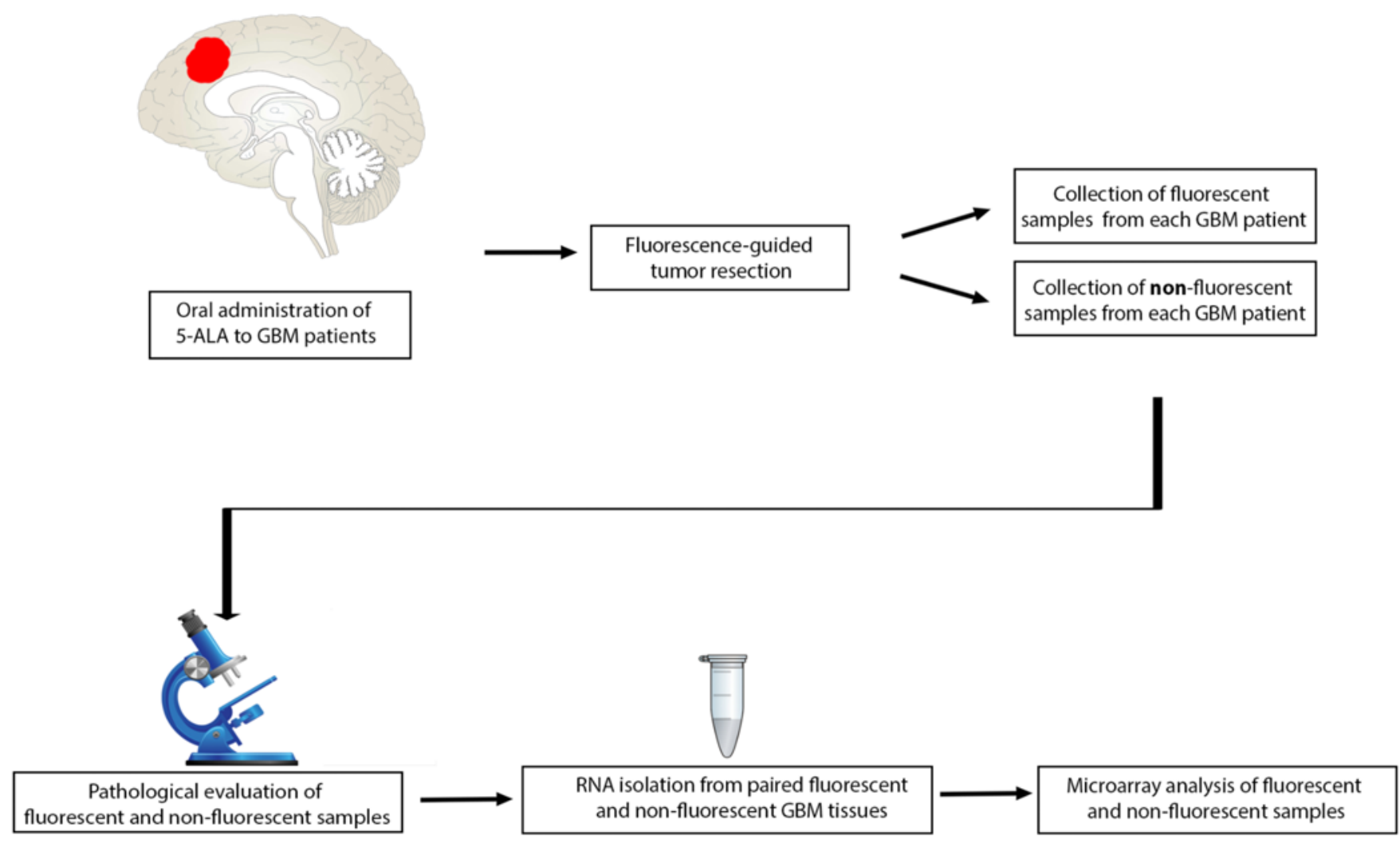

FIG. 1. Schematic representation of the experimental workflow to analyze the pathologic grade and mRNA expression in fluorescent and nonfluorescent GBM-derived tissue.

PCs derived from the centroid values and the microarray data from fluorescent and nonfluorescent samples (see Fig. 2). A cutoff ( $\log 2$ fold change $p<0.1)$ was set to define differential gene expression given the small sample size of this study. A validation experiment, which was performed using samples from 4 different patients and an independent microarray platform, provided additional confidence in our analysis. ${ }^{20}$

We generated signature scores of the samples for each of The Cancer Genome Atlas (TCGA) GBM patients utilizing the Cox proportional hazards model as previously described. ${ }^{1}$ Scores of less than 0 for either the fluorescent or nonfluorescent signatures were categorized as "low" and scores greater than 0 were categorized as "high." To generate the fluorescence scores utilized in Fig. 3 and Supplementary Fig. 2, the microarray profiles of fluorescent and nonfluorescent samples were compared to one another by taking the $\log 2$ fold change of all genes in the array. A $p$ value was computed for each gene and $-\log 10$ transformed. These values were then used to generate fluorescence and nonfluorescence signatures and scores as previously described. ${ }^{2}$

\section{Results}

\section{Intraoperative 5-ALA-Induced Fluorescence in GBM Specimens Is Associated With a Distinct mRNA Expression Profile}

To better understand the biological basis for the het- erogeneity of 5-ALA-induced PpIX fluorescence in GBM, both fluorescent and nonfluorescent GBM specimens were collected from surgical patients undergoing 5-ALAinduced PpIX fluorescence-guided surgery following our previously published methodology. ${ }^{35,37,40}$ Tissue specimens were examined using microarray technology (Fig. 1). Both fluorescent and nonfluorescent specimens represented viable tissue with similar histological appearance (Fig. 2). Specimens with large areas of necrosis were excluded from analysis. As would be expected, microarray expression profiles of high-grade glioma specimens were associated with poor patient survival regardless of their fluorescence status (Fig. 3). We derived a signature of fluorescence and a signature of nonfluorescence utilizing the most upregulated genes in fluorescent and nonfluorescent samples, respectively, as described in Methods. We then used these molecular signatures to derive fluorescence and nonfluorescence "scores" for each of the patients diagnosed with GBM in TCGA database as we have previously reported. ${ }^{1}$ We found that both signatures were associated with decreased survival in TCGA patients (Fig. 3A and B) without a significant overall survival difference between fluorescence groups (Fig. 3C). These observations are consistent with the fact that fluorescent and nonfluorescent GBM specimens in this study were extracted from tumor regions of equivalent pathologic grade (Fig. 2).

The gene expression profiles from fluorescent and nonfluorescent GBM specimens were markedly different (Fig. 4, Supplementary Tables 1 and 2). Tissue with 


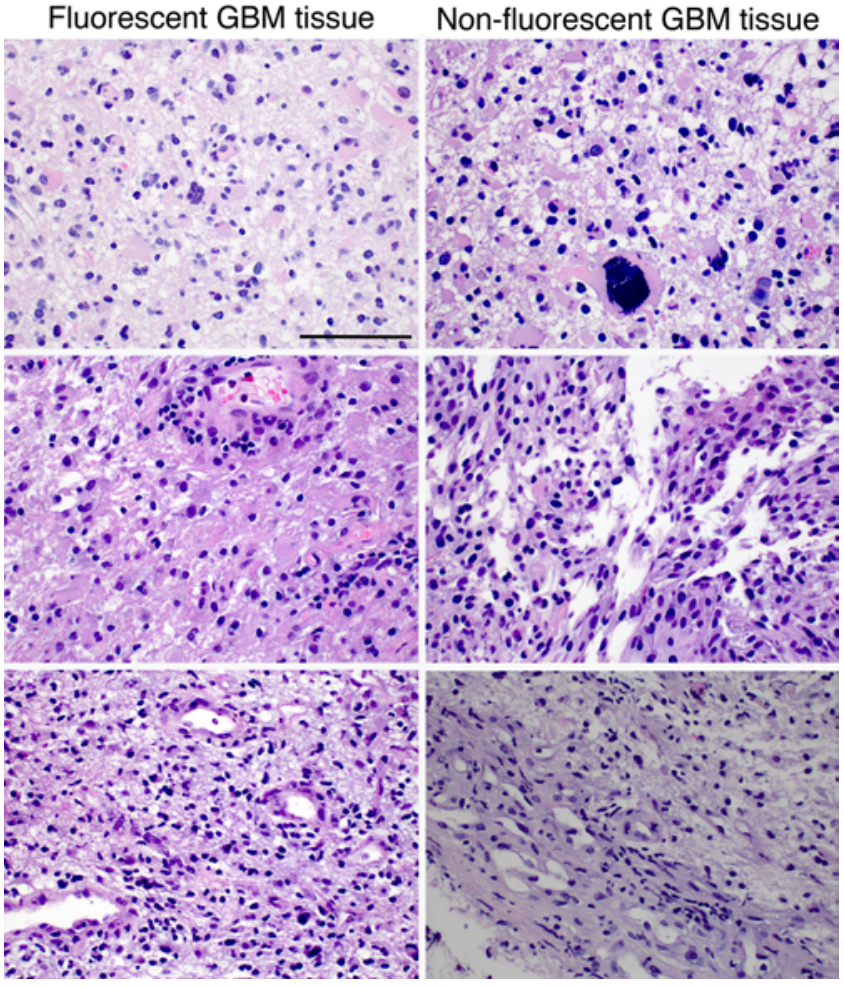

FIG. 2. Microscopy images of fluorescent and nonfluorescent GBM specimens. Representative high-power $\mathrm{H}$ \& $\mathrm{E}$ images from 3 cases with biopsies from fluorescent and nonfluorescent resection areas. Each row represents a different case. Bar $=100 \mu \mathrm{m}$, original magnification $\times 400$.

visible fluorescence expressed higher levels of genes such as IGFBP2, CHI3L1, COL4A1, and VGF that are associated with growth, survival, or angiogenesis (Fig. 4A and
B), ${ }^{7,14,43,46}$ In contrast, nonfluorescent samples expressed genes associated with mature cells of the central nervous system such as MOBP, MAL, and OPALIN (Fig. 4A and C) ${ }^{4,9,11}$ Importantly, expression of genes in the PpIX biosynthetic pathway between fluorescent and nonfluorescent GBM samples was not significantly different (Supplementary Table 3 ).

IPA and GSEA of the gene expression patterns from fluorescent and nonfluorescent GBM specimens revealed that they were associated with distinct cellular functions and biological pathways (Fig. 4D and E, Supplementary Fig. 1A-F). ${ }^{18,32}$ Interestingly, nonfluorescent GBM specimens expressed genes associated with IPA cellular functions characteristic of the neural subtype of GBM such as neural development, neuritogenesis, and neurotransmission (Fig. 4E) ${ }^{42}$ Consistent with this finding, the gene expression profile of nonfluorescent GBM specimens was also enriched for genes associated with GO pathways such as neuron projection development and synaptic transmission (Supplementary Fig. 1D and F). ${ }^{3,18}$ The neural subtype of GBM is the subtype that has a gene expression pattern most similar to the pattern observed in normal brain tissue such as mature neurons and oligodendrocytes. ${ }^{42}$

The gene expression profile of fluorescent GBM specimens, however, was not enriched for genes associated with any one specific molecular subgroup of $\mathrm{GBM}^{42}$ (Fig. 4D, Supplementary Fig. 1A-C). The fluorescent specimens expressed genes associated with cellular functions shared by all subgroups of GBM such as proliferation, survival, and invasion (Fig. 4D). Similarly, GSEA revealed that the gene expression profile of fluorescent specimens was enriched for genes in GO and BIOCARTA pathways characteristic of the proneural, mesenchymal, and classical subtypes of GBM such as NF- $\kappa$ B signaling, TNFR1 signaling, and Notch signaling (Supplementary Fig. 1A-C)., ${ }^{3,812,24,26,42}$ To-
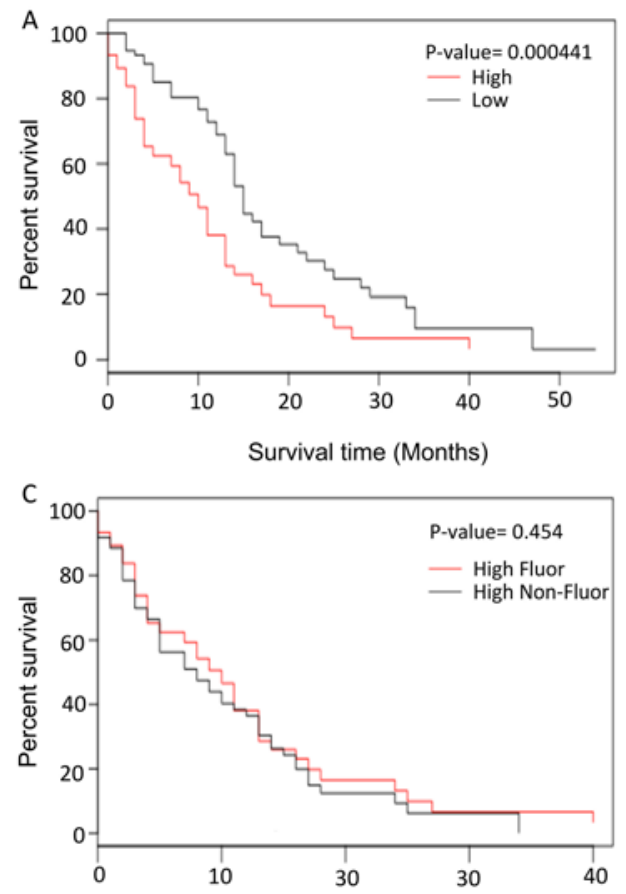

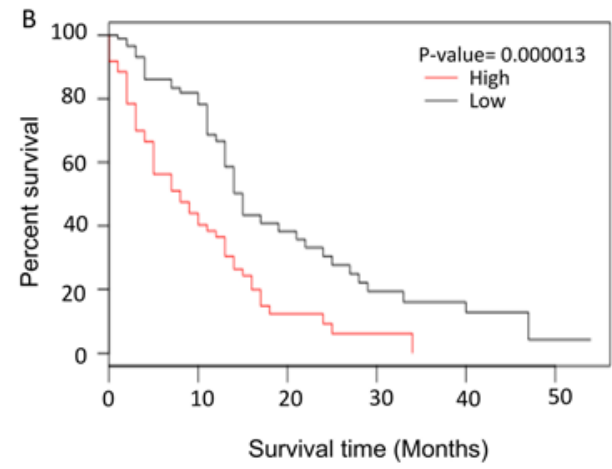

FIG. 3. Signatures derived from top upregulated genes in fluorescent and nonfluorescent samples are associated with decreased survival in GBM patients. A: Kaplan-Meier survival curves comparing TCGA GBM patients with high fluorescent signature scores (High) and low fluorescent signature scores (Low). Log-rank test, $p=0.000441$. B: Kaplan-Meier survival curves comparing GBM patients with high nonfluorescent signature scores and low nonfluorescent signature scores. Log-rank test, $p=0.000013$. C: Kaplan-Meier survival curves comparing GBM patients with high fluorescent signature scores (High Fluor) and GBM patients with high nonfluorescent signature scores (High Non-Fluor). Log-rank test, $p=0.454$. 


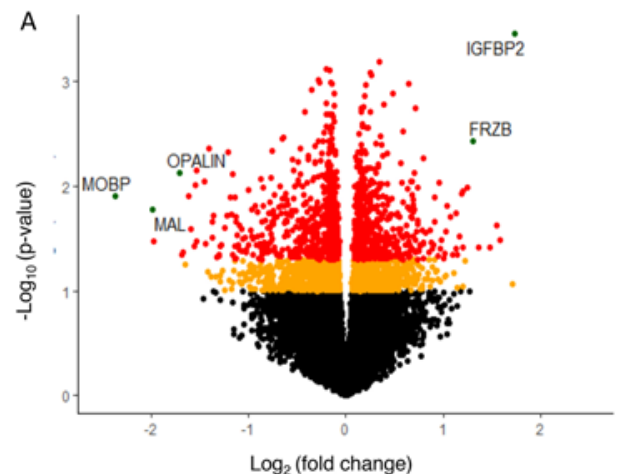

D

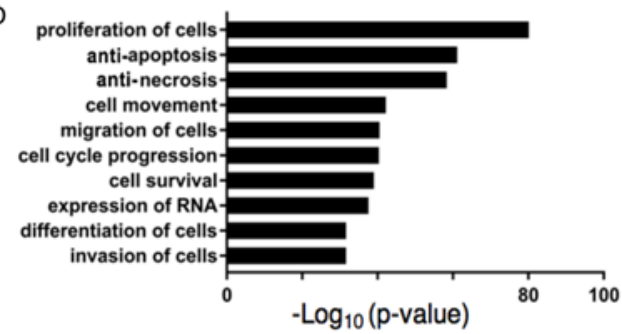

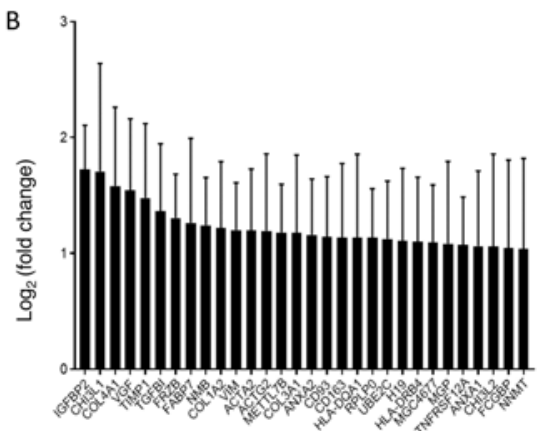

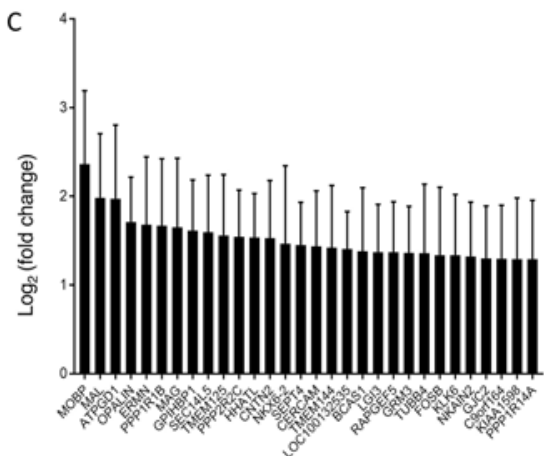

$\mathrm{E}$

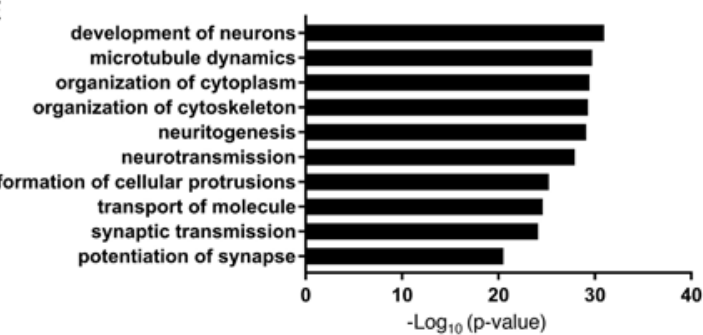

FIG. 4. Intraoperative 5-ALA-induced fluorescence is associated with a distinct mRNA expression profile in human GBM specimens. A: Volcano plot of differentially expressed genes in fluorescent GBM specimens in comparison with nonfluorescent GBM specimens expressed as $\log 2$ fold change. Green dots represent genes whose log2 fold change values are at least 2 with a statistical significance of $p<0.02$. Red dots represent genes whose log2 fold change values have a statistical significance of $p<0.05$. Orange dots represent genes whose log2 fold change values have a statistical significance of $0.05<p<0.1$. Black dots represent genes whose log2 fold change values have a statistical significance of $p<0.1$. B: The 30 most highly expressed genes in fluorescent human GBM specimens when compared with nonfluorescent human GBM specimens. Data represent the log2 fold change of fluorescent GBM specimens $(n=9)$ relative to nonfluorescent specimens $(n=9) \pm$ SEM. C: The 30 most highly expressed genes in nonfluorescent human GBM specimens when compared with the gene expression found in fluorescent human GBM specimens. Data represent the log2 fold change of nonfluorescent GBM specimens $(n=9)$ relative to fluorescent specimens $(n=9) \pm$ SEM. $D$ : The top 10 cellular functions associated with genes significantly upregulated $(p<0.05)$ in fluorescent GBM specimens when compared with nonfluorescent GBM specimens as determined by IPA ${ }^{18} \mathrm{E}$ : Top 10 cellular functions associated with genes significantly upregulated $(p<0.05)$ in nonfluorescent GBM specimens when compared with fluorescent GBM specimens as determined by IPA.

gether, these data demonstrate that fluorescent and nonfluorescent specimens have markedly distinct genetic profiles.

\section{The Gene Expression Profiles of Nonfluorescent GBM Specimens Cluster Around the Neural Subtype of Glioma}

To further characterize the genetic profile of fluorescent and nonfluorescent GBM specimens, we performed a PCA of the gene expression profile from 20 fluorescent and nonfluorescent GBM surgical specimens along with the molecular signatures of the 4 subtypes of GBM: classical, mesenchymal, neural, and proneural (Fig. 5A). To perform this analysis, the gene expression levels of these GBM specimens and the centroid values of the molecular signatures corresponding to each of the 4 subtypes of GBM were transformed into PCs. ${ }^{42}$ In Fig. 5A, we plotted these data utilizing the top 3 PCs derived from this analysis. We observed that the gene expression profiles of the nonfluorescent GBM specimens clustered around the neural subtype of glioma, while those of the fluorescent GBM specimens did not. To validate this finding, we replicated this experiment with microarray expression data from 4 different patients that were obtained using an independent microarray platform (Fig. 5B). In Fig. 5B, we confirmed that the expression profile of the nonfluorescent GBM specimens most closely resembles the neural subtype of glioma.

To corroborate our findings in a larger cohort of patients, we developed a fluorescence signature from the differential gene expression profile between the fluorescent and nonfluorescent GBM specimens. We then used this molecular signature to derive a fluorescence score for each of the neural and nonneural GBM samples in TCGA database as indicated in Methods. We observed that human GBM samples in TCGA database that belonged to the neural subgroup of GBM exhibited significantly lower fluorescence scores than GBM samples that belonged to the other subgroups (Fig. 5C). These data demonstrate that fluorescent and nonfluorescent GBM tissues express distinct gene patterns.

Interestingly, GBM samples from TCGA database exhibited mutations in specific genes based on their fluorescence score. Tumors with mutations in the ABCC9, a protein of the $\mathrm{ABC}$ transporter family responsible for the efflux of 5-ALA,,$^{13}$ had significantly higher fluorescence scores $(p=0.0086)$ consistent with the possibility that the ABCC9 transporter could be playing a role in 5-ALA-induced PpIX fluorescence in GBM. Tumors with mutations 

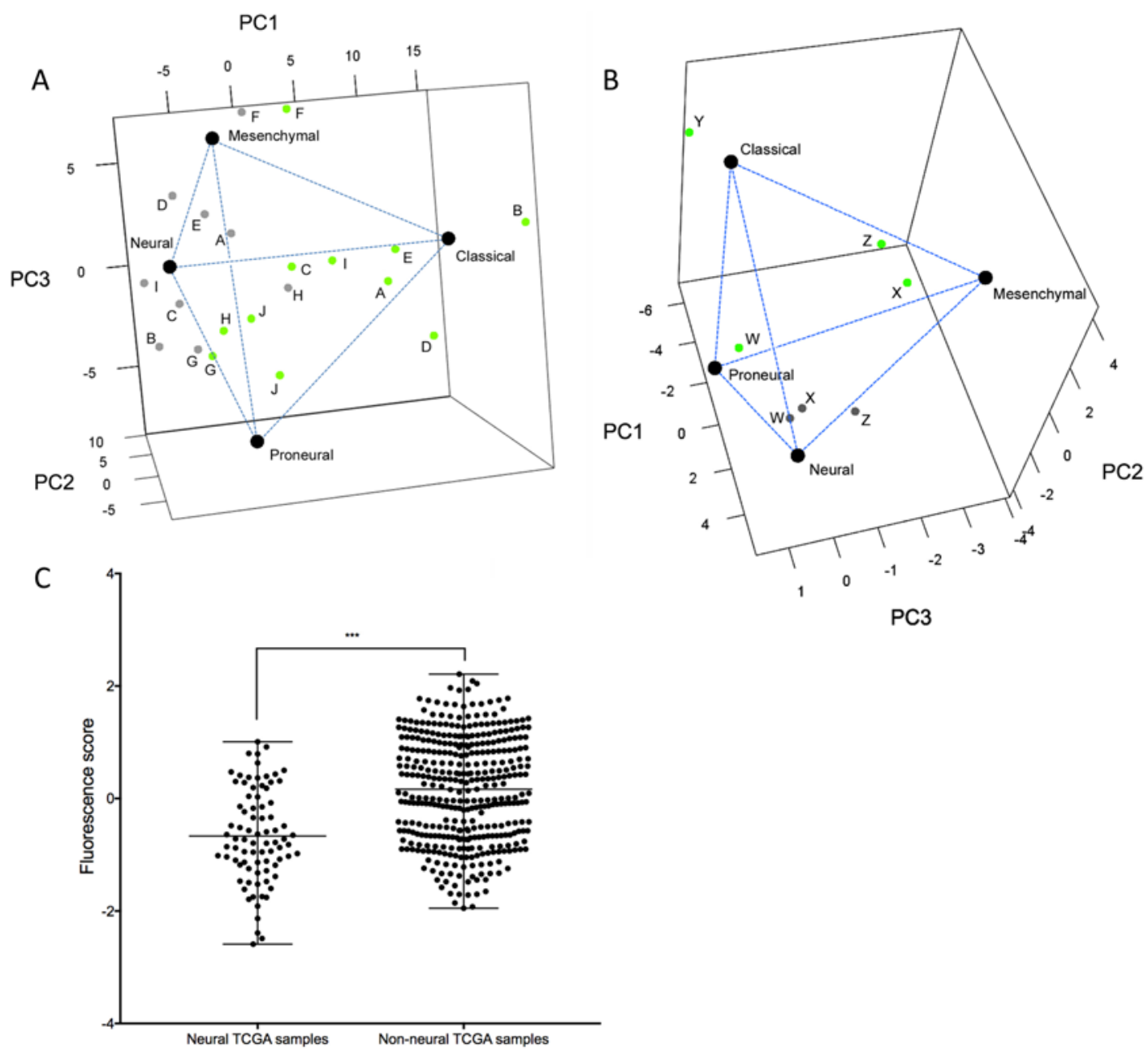

PC3

FIG. 5. Molecular profile of nonfluorescence GBM tissue most closely resembles the neural subtype of glioma. A: PCA plot of GBM molecular subtypes and GBM specimens. GBM subtype centroids corresponding to the classical, mesenchymal, neural, and proneural subtypes of GBM are illustrated as black dots. Letters $A-J$ denote the 10 different human tumors analyzed. GBM specimens that fluoresced after 5-ALA administration are illustrated as green dots, and GBM specimens that did not fluoresce after 5-ALA administration are illustrated as gray dots. B: Confirmatory PCA plot of GBM molecular subtypes corresponding to 7 human GBM specimens. GBM subtype centroids corresponding to the classical, mesenchymal, neural, and proneural subtypes of GBM are illustrated as black dots. Letters $W-Z$ denote the 4 different human tumors analyzed. GBM specimens that fluoresce after 5-ALA administration are illustrated as green dots, and GBM specimens that do not fluoresce after 5-ALA administration are illustrated as gray dots. C: Fluorescence score of GBM patients from TCGA, as determined by our fluorescence signature, subdivided as either neural or nonneural. Data represent $600 \mathrm{GBM}$ patient samples presented as the mean $\pm \mathrm{SD},{ }^{* * *} \mathrm{p}<0.001$.

in IDH1 and ATRX had significantly lower fluorescent scores ( $p=0.00038$ and $p=0.00076$, respectively) (Supplementary Fig. 2) ${ }^{6,17,23}$ These observations further reveal the distinctions between fluorescent and nonfluorescent GBM tissues.

\section{Discussion}

GBM is the most common and aggressive primary brain tumor in adults. Despite optimal surgical and medical therapy, recurrence of disease typically develops adjacent to the resection margins due to residual microscopic infiltrating glioma cells that were not resected or eradicated after radiation or chemotherapy. 5-ALA-induced PpIX fluorescence-guided resection has been shown to improve GTR and 6-month progression-free survival in patients. ${ }^{10,29} \mathrm{How}-$ ever, the efficacy of this technique in GBM has been compromised by the lack of visible 5-ALA-induced PpIX fluorescence in histopathologically confirmed tumor tissue..$^{16,25}$, 36,37,44 A better understanding of the biological basis for this heterogeneity in 5-ALA-induced PpIX tumor fluorescence might contribute to improving intraoperative detection of malignant tissue and decreasing the rate of glioma recurrence.

Our findings indicate that tissues taken from different areas of the same GBM tumor exhibit different levels of 5-ALA (fluorescence vs nonfluorescence) and different patterns of gene expressions measured at the transcriptional level. These observations suggest a molecular basis for the 
heterogeneity in 5-ALA-induced fluorescence observed in different GBM tumors and within individual tumors. Consistent with this notion, nonfluorescent regions were observed to more closely resemble the neural subgroup of GBM.

Understanding of those factors responsible for falsenegative fluorescence imaging and the suboptimal predictive value of tissue that does not fluoresce is incomplete. Surgeons should be aware that a lack of fluorescence in high-grade glioma may result not only from tumor necrosis, an intact blood-brain barrier, low levels of tumor cell invasion, or lower-grade tumor, but also from molecular subtypes of high-grade tumor being present within an individual tumor. PpIX fluorescence is not just a function of PpIX tissue levels, but it also reflects tissue architecture and constituents. ${ }^{22,37,39-41}$ Variable optical properties of tissues may "mask" PpIX fluorescence. These optical properties are themselves determined by factors such as cellularity, necrosis, oxygenation, vascularity, and hemoglobin, among other factors. Our work provides an additional determinant, namely, cellular variability based on different transcriptional patterns that characterize GBM subtypes.

Roberts and colleagues previously showed that using a multiparametric approach that detects not only PpIX but additional biomarkers using a quantitative optical probe significantly improved diagnostic accuracy (e.g., improved sensitivity and negative predictive value) not just in highgrade gliomas but also in other tumor subtypes such as low-grade gliomas. ${ }^{38}$ Since GBM is very heterogeneous, often containing different glioma molecular subtypes (neural, proneural, mesenchymal, or classical) within the same tumor, an approach using multiple fluorophore and optical biomarkers may be more effective in detecting these highly heterogeneous tumors intraoperatively. Combining 2 or more different fluorescent biomarkers for intraoperative detection methods, such as ALA, fluorescein, antiEGFR or anti-VEGFR targeted fluorophores, quantum dot nanoparticles, or other agents, one could potentially target different subtypes of GBM simultaneously ${ }^{27,39}$ and thereby improve intraoperative tumor detection and GTR. To this end, an improved molecular understanding will help guide further technological developments to advance the field of fluorescence-guided neurosurgical oncology.

\section{Conclusions}

In this study, we found that fluorescent and nonfluorescent GBM surgical specimens expressed distinct genetic expression profiles (Figs. 3-5). The expression profile of nonfluorescent tumor tissue was enriched for genes associated with cellular functions and biological pathways characteristic of the neural subgroup of GBM (Figs. 3 and 4). Furthermore, PCA revealed that the expression profiles of nonfluorescent tumor tissues most closely resemble the neural subgroup of GBM (Fig. 5A). Consistent with these observations, human GBM samples from TCGA database that belonged to the neural subgroup of GBM were identified to have lower fluorescence scores than samples that belonged to other subgroups of GBM (Fig. 5B).

In a manner consistent with the high degree of heterogeneity observed in high-grade gliomas, ${ }^{28}$ our results dem- onstrate that sections within glioma tumors that respond differently to 5-ALA are molecularly distinct. These findings are consistent with previous findings demonstrating differences in individual gene expression associated with 5-ALA-induced fluorescence in GBM. ${ }^{33,34,47}$ Furthermore, these findings are consistent with other work using quantitative technologies, which highlights the importance of tissue constituents and their effects on the detected PpIX fluorescence..$^{38}$ That work provides further rationale to develop novel technologies for improved detection of PpIX fluorescence and intrinsic tissue properties toward a multiparametric approach for fluorescence-guided surgery as well as help explore combinations of fluorescence agents such as fluorescein, quantum dot nanoparticles, anti-EGFR, or anti-VEGFR antibody-bound fluorophores that could potentially target different molecular subtypes of GBM. ${ }^{27}$

\section{Acknowledgments}

Support was generously provided by National Institutes of Health Grant No. R01NS052274 (D.W. Roberts), the Theodora B. Betz Foundation (M.A. Israel), the Jordan and Kyra Memorial Foundation (M.A. Israel), and the Andrea Clark Nelson Medical Research Endowment (M.A. Israel).

\section{References}

1. Almiron Bonnin DA, Havrda MC, Lee MC, Liu H, Zhang Z, Nguyen LN, et al: Secretion-mediated STAT3 activation promotes self-renewal of glioma stem-like cells during hypoxia. Oncogene 37:1107-1118, 2018

2. Almiron Bonnin DA, Ran C, Havrda MC, Liu H, Hitoshi Y, Zhang Z, et al: Insulin-mediated signaling facilitates resistance to PDGFR inhibition in proneural hPDGFB-driven gliomas. Mol Cancer Ther 16:705-716, 2017

3. Ashburner M, Ball CA, Blake JA, Botstein D, Butler H, Cherry JM, et al: Gene ontology: tool for the unification of biology. Nat Genet 25:25-29, 2000

4. Auvergne RM, Sim FJ, Wang S, Chandler-Militello D, Burch J, Al Fanek Y, et al: Transcriptional differences between normal and glioma-derived glial progenitor cells identify a core set of dysregulated genes. Cell Reports 3:2127-2141, 2013

5. Brown TJ, Brennan MC, Li M, Church EW, Brandmeir NJ, Rakszawski KL, et al: Association of the extent of resection with survival in glioblastoma: a systematic review and metaanalysis. JAMA Oncol 2:1460-1469, 2016

6. Cohen AL, Holmen SL, Colman H: IDH1 and IDH2 mutations in gliomas. Curr Neurol Neurosci Rep 13:345-345, 2013

7. Cohen N, Shani O, Raz Y, Sharon Y, Hoffman D, Abramovitz $\mathrm{L}$, et al: Fibroblasts drive an immunosuppressive and growthpromoting microenvironment in breast cancer via secretion of Chitinase 3-like 1. Oncogene 36:4457-4468, 2017

8. Cooper LAD, Gutman DA, Long Q, Johnson BA, Cholleti SR, Kurc T, et al: The proneural molecular signature is enriched in oligodendrogliomas and predicts improved survival among diffuse gliomas. PLoS One 5:e12548, 2010

9. Dugas JC, Tai YC, Speed TP, Ngai J, Barres BA: Functional genomic analysis of oligodendrocyte differentiation. J Neurosci 26:10967-10983, 2006

10. Eljamel S: 5-ALA fluorescence image guided resection of glioblastoma multiforme: a meta-analysis of the literature. Int J Mol Sci 16:10443-10456, 2015

11. Golan N, Adamsky K, Kartvelishvily E, Brockschnieder D, Möbius W, Spiegel I, et al: Identification of Tmem10/Opalin as an oligodendrocyte enriched gene using expression profiling combined with genetic cell ablation. Glia 56:1176-1186, 2008 
12. Goldhoff P, Clarke J, Smirnov I, Berger MS, Prados MD, James CD, et al: Clinical stratification of glioblastoma based on alterations in retinoblastoma tumor suppressor protein (RB1) and association with the proneural subtype. J Neuropathol Exp Neurol 71:83-89, 2012

13. Hagiya Y, Endo Y, Yonemura Y, Takahashi K, Ishizuka M, Abe F, et al: Pivotal roles of peptide transporter PEPT1 and ATP-binding cassette (ABC) transporter ABCG2 in 5-aminolevulinic acid (ALA)-based photocytotoxicity of gastric cancer cells in vitro. Photodiagn Photodyn Ther 9:204-214, 2012

14. Jin R, Shen J, Zhang T, Liu Q, Liao C, Ma H, et al: The highly expressed COL4A1 genes contributes to the proliferation and migration of the invasive ductal carcinomas. Oncotarget 8:58172-58183, 2017

15. Kesari S, Rahme GJ, Almiron DA, Israel MA: Brain tumors, in Schwab M (ed): Encyclopedia of Cancer. Berlin: Springer, 2017

16. Kim A, Roy M, Dadani F, Wilson BC: A fiberoptic reflectance probe with multiple source-collector separations to increase the dynamic range of derived tissue optical absorption and scattering coefficients. Opt Express 18:5580-5594, 2010

17. Koschmann C, Lowenstein PR, Castro MG: ATRX mutations and glioblastoma: Impaired DNA damage repair, alternative lengthening of telomeres, and genetic instability. Mol Cell Oncol 3:e1167158, 2016

18. Krämer A, Green J, Pollard J Jr, Tugendreich S: Causal analysis approaches in Ingenuity Pathway Analysis. Bioinformatics 30:523-530, 2014

19. Lê S, Josse J, Husson F: FactoMineR: An R package for multivariate analysis. J Stat Softw 25:18, 2008

20. Li Y, Byun J, Cai G, Xiao X, Han Y, Cornelis O, et al: FastPop: a rapid principal component derived method to infer intercontinental ancestry using genetic data. BMC Bioinformatics 17:122, 2016

21. Moiyadi A, Syed P, Srivastava S: Fluorescence-guided surgery of malignant gliomas based on 5-aminolevulinic acid: paradigm shifts but not a panacea. Nat Rev Cancer 14:146, 2014

22. Montcel B, Mahieu-Williame L, Armoiry X, Meyronet D, Guyotat J: Two-peaked 5-ALA-induced PpIX fluorescence emission spectrum distinguishes glioblastomas from low grade gliomas and infiltrative component of glioblastomas. Biomed Opt Express 4:548-558, 2013

23. Nandakumar P, Mansouri A, Das S: The role of ATRX in glioma biology. Front Oncol 7:236, 2017

24. Nishimura D: BioCarta. Biotech Softw Internet Rep 2:117120,2001

25. Roberts DW, Valdés PA, Harris BT, Fontaine KM, Hartov A, Fan X, et al: Coregistered fluorescence-enhanced tumor resection of malignant glioma: relationships between $\delta$-aminolevulinic acid-induced protoporphyrin IX fluorescence, magnetic resonance imaging enhancement, and neuropathological parameters. Clinical article. J Neurosurg 114:595-603, 2011

26. Saito N, Fu J, Zheng S, Yao J, Wang S, Liu DD, et al: A high Notch pathway activation predicts response to $\gamma$ secretase inhibitors in proneural subtype of glioma tumor-initiating cells. Stem Cells 32:301-312, 2014

27. Senders JT, Muskens IS, Schnoor R, Karhade AV, Cote DJ, Smith TR, et al: Agents for fluorescence-guided glioma surgery: a systematic review of preclinical and clinical results. Acta Neurochir (Wien) 159:151-167, 2017

28. Soeda A, Hara A, Kunisada T, Yoshimura S, Iwama T, Park DM: The evidence of glioblastoma heterogeneity. Sci Rep 5:7979, 2015

29. Stummer W, Pichlmeier U, Meinel T, Wiestler OD, Zanella F, Reulen HJ: Fluorescence-guided surgery with 5-aminolevulinic acid for resection of malignant glioma: a randomised controlled multicentre phase III trial. Lancet Oncol 7:392 401, 2006

30. Stupp R, Hegi ME, Mason WP, van den Bent MJ, Taphoorn MJ, Janzer RC, et al: Effects of radiotherapy with concomitant and adjuvant temozolomide versus radiotherapy alone on survival in glioblastoma in a randomised phase III study: 5-year analysis of the EORTC-NCIC trial. Lancet Oncol 10:459-466, 2009

31. Stupp R, Taillibert S, Kanner A, Read W, Steinberg D, Lhermitte B, et al: Effect of tumor-treating fields plus maintenance temozolomide vs maintenance temozolomide alone on survival in patients with glioblastoma: a randomized clinical trial. JAMA 318:2306-2316, 2017

32. Subramanian A, Tamayo P, Mootha VK, Mukherjee S, Ebert BL, Gillette MA, et al: Gene set enrichment analysis: a knowledge-based approach for interpreting genome-wide expression profiles. Proc Natl Acad Sci U S A 102:1554515550,2005

33. Suzuki T, Wada S, Eguchi H, Adachi J, Mishima K, Matsutani M, et al: Cadherin 13 overexpression as an important factor related to the absence of tumor fluorescence in 5-aminolevulinic acid-guided resection of glioma. J Neurosurg 119:1331-1339, 2013

34. Teng L, Nakada M, Zhao SG, Endo Y, Furuyama N, Nambu E, et al: Silencing of ferrochelatase enhances 5-aminolevulinic acid-based fluorescence and photodynamic therapy efficacy. Br J Cancer 104:798-807, 2011

35. Valdes PA, Angelo JP, Choi HS, Gioux S: qF-SSOP: realtime optical property corrected fluorescence imaging. Biomed Opt Express 8:3597-3605, 2017

36. Valdés PA, Fan X, Ji S, Harris BT, Paulsen KD, Roberts DW: Estimation of brain deformation for volumetric image updating in protoporphyrin IX fluorescence-guided resection. Stereotact Funct Neurosurg 88:1-10, 2010

37. Valdés PA, Jacobs V, Harris BT, Wilson BC, Leblond F, Paulsen KD, et al: Quantitative fluorescence using 5-aminolevulinic acid-induced protoporphyrin IX biomarker as a surgical adjunct in low-grade glioma surgery. J Neurosurg 123:771-780, 2015

38. Valdés PA, Kim A, Leblond F, Conde OM, Harris BT, Paulsen KD, et al: Combined fluorescence and reflectance spectroscopy for in vivo quantification of cancer biomarkers in low- and high-grade glioma surgery. J Biomed Opt 16:116007, 2011

39. Valdés PA, Leblond F, Jacobs VL, Wilson BC, Paulsen KD, Roberts DW: Quantitative, spectrally-resolved intraoperative fluorescence imaging. Sci Rep 2:798, 2012

40. Valdés PA, Leblond F, Kim A, Harris BT, Wilson BC, Fan $X$, et al: Quantitative fluorescence in intracranial tumor: implications for ALA-induced PpIX as an intraoperative biomarker. J Neurosurg 115:11-17, 2011

41. Valdés PA, Roberts DW, Lu FK, Golby A: Optical technologies for intraoperative neurosurgical guidance. Neurosurg Focus 40(3):E8, 2016

42. Verhaak RGW, Hoadley KA, Purdom E, Wang V, Qi Y, Wilkerson MD, et al: Integrated genomic analysis identifies clinically relevant subtypes of glioblastoma characterized by abnormalities in PDGFRA, IDH1, EGFR, and NF1. Cancer Cell 17:98-110, 2010

43. Wang X, Prager BC, Wu Q, Kim LJY, Gimple RC, Shi Y, et al: Reciprocal signaling between glioblastoma stem cells and differentiated tumor cells promotes malignant progression. Cell Stem Cell 22:514-528.e5, 2018

44. Whitson WJ, Valdes PA, Harris BT, Paulsen KD, Roberts DW: Confocal microscopy for the histological fluorescence pattern of a recurrent atypical meningioma: case report. Neurosurgery 68:E1768-E1773, 2011

45. Widhalm G: Intra-operative visualization of brain tumors with 5-aminolevulinic acid-induced fluorescence. Clin Neuropathol 33:260-278, 2014 
46. Yau SW, Azar WJ, Sabin MA, Werther GA, Russo VC: IGFBP-2-taking the lead in growth, metabolism and cancer. J Cell Commun Signal 9:125-142, 2015

47. Zhao SG, Chen XF, Wang LG, Yang G, Han DY, Teng L, et al: Increased expression of $\mathrm{ABCB} 6$ enhances protoporphyrin IX accumulation and photodynamic effect in human glioma. Ann Surg Oncol 20:4379-4388, 2013

\section{Disclosures}

Dr. Valdes: patent holder with Dartmouth College. Dr. Paulsen: ownership in InSight Surgical Technologies, LLC, and CairnSurgical; and patent holder with Dartmouth. Dr. Roberts: clinical or research support for the study described from DUSA Pharmaceutical, Inc., Carl Zeiss Meditec, Inc., and Medtronic Navigation; ownership in InSight Surgical Technologies, LLC; and patent holder with Dartmouth.

\section{Author Contributions}

Conception and design: Israel, Almiron Bonnin, Havrda, Ran, Valdes, Harris, Paulsen, Roberts. Acquisition of data: Israel, Almiron Bonnin, Lee, Evans, Ran, Valdes, Harris, Paulsen, Roberts. Analysis and interpretation of data: Israel, Almiron Bonnin, Lee, Evans, Ran, Qian, Harrington, Valdes, Cheng, Amos,
Harris, Paulsen, Roberts. Drafting the article: Israel, Almiron Bonnin, Havrda, Evans, Roberts. Critically revising the article: Israel, Almiron Bonnin, Havrda, Evans, Qian, Harrington, Valdes, Harris, Roberts. Reviewed submitted version of manuscript: all authors. Approved the final version of the manuscript on behalf of all authors: Israel. Statistical analysis: Israel, Almiron Bonnin, Lee, Ran, Qian, Harrington, Cheng, Amos, Roberts. Administrative/technical/material support: Israel, Almiron Bonnin, Havrda, Harris, Paulsen, Roberts. Study supervision: Israel, Almiron Bonnin, Roberts.

\section{Supplemental Information}

\section{Online-Only Content}

Supplemental material is available with the online version of the article.

Supplementary Figures and Tables. https://thejns.org/doi/ suppl/10.3171/2019.2.JNS183128.

\section{Correspondence}

Mark A. Israel: Norris Cotton Cancer Center at Dartmouth, Lebanon, NH. mark.a.israel@dartmouth.edu. 